\section{Avaliação da situação de saúde por profissionais da atenção primária em saúde com base no georreferencimento dos sistemas de informação}

\author{
Health situation assessment by primary care \\ workers based on geographic information systems
}

\author{
Evaluación de la situación de los profesionales \\ de la salud para la atención primaria de salud, \\ basándose en la georreferenciación de los \\ sistemas de información
}

\author{
1 Faculdade de Medicina, \\ Universidade Federal do Rio \\ Grande do Sul, Porto Alegre, \\ Brasil. \\ 2 Secretaria Municipal de \\ Saúde de Porto Alegre, Porto \\ Alegre, Brasil. \\ 3 Grupo Hospitalar \\ Conceição, Porto Alegre, \\ Brasil. \\ Correspondência \\ F. Ritter \\ Faculdade de Medicina, \\ Universidade Federal do Rio \\ Grande do Sul. \\ Rua General Caldwell 847, \\ apto. 704, Porto Alegre, RS \\ 90130-051, Brasil. \\ ritterfernando@gmail.com
}

\begin{abstract}
Primary healthcare has made little use of information systems to assess the population's health situation due to the difficulty in understanding the reports. Generic definitions of actions are common, based on empirical observations. The current study aimed to evaluate whether the introduction of georeferenced indicators can serve to better identify individuals' health situation, which would help planning actions by health teams. Healthcare workers from eight teams answered a questionnaire at three different moments: the first, before reading the information system's reports; the second after reading; and the third after using georeferencing. The results showed a significant difference in the classification of the health situation using georeferencing when compared to the previous moments ( $p<$ 0.05). Georeferencing facilitated analysis of the health situation, fostering better monitoring of work processes. Finally, use of the data points to rationalization of actions and possible upgrading of healthcare. The study suggests the use of georeferencing in the work agenda to become an effective tool for orienting actions.
\end{abstract}

Family Health; Geographic Information Systems; Health Evaluation
Fernando Ritter 1,2

Roger dos Santos Rosa 1

Rui Flores 2,3

\section{Resumo}

A atenção primária em saúde (APS) pouco tem se valido dos sistemas de informação para avaliar a situação de saúde da população devido à dificuldade de compreensão dos relatórios. É usual a definição genérica das ações a partir de constatações empíricas. O objetivo desse trabalho é avaliar se a introdução de indicadores georreferenciados pode ser uma tecnologia para melhorar a identificação da situação de saúde das pessoas, o que ajudaria no planejamento das ações das equipes. Para tanto, foi aplicado um questionário nos profissionais de oito equipes em três momentos: o primeiro, antes da leitura dos relatórios do sistema de informação, o segundo após a leitura e o terceiro usando os georreferenciados. Os resultados mostraram diferença significativa na classificação da situação de saúde quando da utilização do georreferenciamento comparado aos momentos anteriores $(p<$ 0,05). O georreferenciamento facilitou a análise da situação de saúde, propiciando melhor monitoramento dos processos de trabalho. Por fim, a utilização aponta para uma racionalização das ações e possível qualificação da atenção à saúde. Sugere-se o uso do georreferenciamento na agenda de trabalho para que se tornem uma ferramenta efetiva e norteadora das ações.

Saúde da Família; Sistemas de Informação Geográfica; Avaliação em Saúde 


\section{Introdução}

A atuação territorial e a delimitação geográfica em atenção primária em saúde possibilitam identificar espaços determinados que possam gerar riscos para a saúde da população. A Estratégia Saúde da Família (ESF) permite conhecer características das pessoas e das comunidades, bem como desenvolver programas e projetos específicos frente aos diagnósticos levantados. Nesse contexto, os Sistemas de Informação em Saúde (SIS) têm papel de subsidiar o processo de tomada de decisão, sendo instrumentos que permitem organizar e analisar os dados necessários à definição de problemas e riscos à saúde, bem como avaliar o impacto que os serviços possam ter no estado de saúde da população 1 .

No entanto, o processo decisório local pouco tem se valido dessas informações, contribuindo para a ineficiência da gestão dos recursos pessoais, materiais, financeiros, de equipamentos, medicamentos, tecnologia etc. Os profissionais das equipes da ESF têm o conhecimento da importância dos SIS, no entanto, a sua utilização e o seu envolvimento com os sistemas de informação são esporádicos ${ }^{2}$. Isso porque os profissionais ainda têm embutidos em sua prática a valorização da produção numérica de procedimentos técnicos. Isso contribui com a pouca utilização dos SIS como instrumento de diagnóstico da realidade local, bem como para o planejamento, o monitoramento e a avaliação das atividades desenvolvidas.

A ESF no Brasil tem o objetivo de estruturar a atenção primária em saúde, conforme dispõe a Portaria GM no 2.488/2011 3. Esse novo modelo de atenção primária em saúde define a reorientação do SUS, baseando-se no princípio da descentralização e fundamentalmente no fortalecimento dos níveis periféricos de atenção à saúde 4 . A reorientação possibilita ações com maior autonomia na tomada de decisões em relação às necessidades da população do território de abrangência da Unidade de Saúde da Família.

A territorialização é um passo fundamental para a caracterização da população, bem como para avaliação do impacto do serviço ${ }^{5}$. A partir disso, pode-se considerar como atividade primordial para a consolidação das ações em saúde na ESF: a realização de diagnóstico e análise da situação de saúde, bem como a definição de estratégias necessárias para a superação dos problemas do coletivo das pessoas que fazem parte de cada unidade de saúde. Para a operacionalização de um diagnóstico situacional é necessária a otimização de um SIS que permita aos gestores, aos trabalhadores e à população autonomia suficiente para conhecer com atualidade e pro- fundidade as matérias sobre quais devem agir no contexto da saúde coletiva $6,7,8$.

Na ESF, o instrumento de coleta, armazenamento e processamento de dados é o Sistema de Informação da Atenção Básica (SIAB) 9. Esse sistema propõe-se a (re)organizar os serviços baseado na prática da vigilância em um território determinado, imprimindo qualidade às ações por ser alimentado com dados demográficos, de morbidade referida e de condições sociais locais. Tais dados podem se reverter em indicadores que subsidiem o planejamento, a programação e a tomada de decisão da equipe de forma interdisciplinar. Esse sistema é fundamental por auxiliar no monitoramento e na avaliação das ações e serviços por meio de relatórios que permitem o conhecimento da realidade sócio-sanitária da população 10,11,12.

Para a gestão de sistemas de saúde em atenção primária em saúde é fundamental que os profissionais desenvolvam habilidades para utilizar instrumentos que possibilitem a reflexão crítica e a transformação da sua ação como é caso dos SIS. Assim, poderão provocar mudança no modo como as pessoas lidam com a saúde e a doença. No entanto, na prática das unidades de saúde, as prioridades ou ações estão quase sempre alienadas, fora do poder de decisão dos trabalhadores que realizam as atividades. Quem define as ações ou atividades a serem desenvolvidas são, geralmente, grupos restritos que ocupam níveis mais elevados da hierarquia institucional 13,14,15. Via de regra, objetivos são definidos genericamente, pois surgem de constatações que não expressam a necessidade local.

A partir disso, é necessário que a equipe de saúde domine as tecnologias de informação que possibilitem o desenvolvimento de diagnósticos comunitários mais adequados a sua realidade. É preciso compreender, porque a doença está presente e como pode ser prevenida 7,16. Portanto, a territorialização dos SIS possibilita a localização dos problemas de saúde e a identificação de desigualdades. O território não corresponde apenas a uma delimitação geográfica, mas tem que esquematizar um perfil demográfico, epidemiológico, administrativo, político, social e cultural que o caracteriza e se expressa em um processo de permanente construção ${ }^{17}$. No entanto, existem inúmeras dificuldades para os profissionais fazerem a interpretação dos dados gerados por eles mesmos durante o seu processo de trabalho.

O georreferenciamento é definido como o processo de associação do dado a um mapa e o resultado desse processo é a criação de elementos gráficos que podem ser usados para localizar os determinantes de saúde/doença dos grupos populacionais 18. Os Sistemas de Informação 
Georreferenciados (SIG) trabalham com dados agregados por área, assim, diferentes níveis de taxa podem ser expressos por cores diferentes e níveis semelhantes. Desse modo, constituemse em um instrumento prático para evidenciar as desigualdades existentes num dado território 19,20,21,22.

Para que a ESF se fortaleça e os profissionais sintam-se sujeitos ativos na mudança do modelo de atenção são necessários avaliações e acompanhamentos sistemáticos das ações. No entanto, boa parte dos municípios executa o procedimento de coleta de dados para os SIS de âmbito nacional com a função de cumprir critérios formais de repasse de verbas. Estudo realizado identificou necessidade do planejamento das ações em saúde baseado nas situações de saúde da população ${ }^{23}$. No entanto, as atividades baseavamse em observações pessoais, sem o subsídio de dados epidemiológicos, sociais e econômicos do município.

Por tudo isso, o objetivo desse trabalho é avaliar se a introdução de indicadores de saúde georreferenciados influência na percepção, por parte dos profissionais da ESF, sobre a situação de saúde da população da sua área de abrangência.

\section{Metodologia}

O estudo foi desenvolvido com os profissionais que fazem parte de equipes da ESF de uma das oito Gerências Distritais (GD) do Município de Porto Alegre (Rio Grande do Sul), a GD Sul/ Centro-Sul (Figura 1). Essa GD é constituída por seis unidades da ESF da modalidade simples e duas da modalidade dupla, sendo responsável por 26.173 habitantes, conforme dados do SIAB. Aceitaram participar da pesquisa oito médicos, seis enfermeiros, 13 técnicos de enfermagem e 23 agentes comunitários de saúde (ACS). A coleta de dados ocorreu em três momentos distintos por meio de um questionário estruturado e entregue, individualmente, aos sujeitos de pesquisa em reuniões de equipes que ocorrem semanalmente. Para responder, esses tiveram a sua disposição uma escala ordinal do tipo Likert para avaliar a percepção com relação à situação de saúde da população que integra a área de abrangência das unidades da ESF 24. Os valores estabelecidos nos graus dessa escala foram: ruim (1), regular (2), bom (3), muito bom (4) e excelente (5), sendo que 24 perguntas foram feitas da seguinte forma: "A partir dos seus conhecimentos prévios e sua atuação prática profissional, como você classificaria a atuação da equipe com relação ao cuidado das gestantes acompanhadas no mês?" Ou ainda: "Como você classificaria a prevalência esperada de pessoas hipertensas cadastradas em relação ao número de pessoas de 20 anos ou mais?".

Para tanto, focou-se em dados contidos no relatório SSA2 (Secretaria Municipal de Saúde de Porto Alegre. Situação de saúde e o acompanhamento das famílias. 18/Jan/2010) do SIAB de janeiro a dezembro de 2009 (Tabela 1). No momento I, os profissionais responderam as perguntas a partir dos seus conhecimentos prévios e da sua atuação prática profissional. No momento II, receberam o mesmo questionário e classificaram a situação de saúde tendo como base o relatório SSA2 e ainda foram acrescidas perguntas que avaliaram qual a interação de cada um dos profissionais com o SIAB no que se refere à acessibilidade, à apresentação, à compreensibilidade e à importância da sua utilização no processo de trabalho da equipe. Para ver essa interação com o sistema informação, mais uma vez foi usada uma escala ordinal do tipo Likert de cinco pontos que avaliou qual o grau dessa interação. No momento III, os profissionais receberam o relatório SSA2 georreferenciado (SSA2g), ou seja, na forma de mapas e divididos por microárea de ACS e responderam o terceiro questionário com as mesmas perguntas dos momentos I e II.

Os mapas expressavam os mesmos indicadores de saúde, os quais apresentam a situação de saúde da população, separados por uma graduação de cinco tons de cinza. Essa graduação classificou a situação de saúde das pessoas que fazem parte em cada microárea, conforme se observa na Figura 2. Os pontos branco ou preto referemse à localização espacial das unidades de saúde. Os dados foram apropriados no pacote estatístico SPSS (SPSS Inc., Chicago, Estados Unidos). Foram realizadas análises descritivas dos dados e a associação estatística foi buscada através da comparação de medidas nos três momentos da pesquisa, como indica Field 25 , por meio da análise de variância, conhecida como ANOVA, sendo que as categorias da escala Likert tinham valores de um a cinco. Foi utilizado um valor de alfa igual a 0,05. Esse estudo foi submetido e aprovado no Comitê de Ética do Grupo Hospitalar Conceição de Porto Alegre.

\section{Resultados}

Houve a perda de quatro profissionais do momento I para o II e mais dois entre o momento II e o III. A Tabela 2 apresenta os resultados dos valores médios e desvio-padrão para cada indicador que apresentou diferenças estatisticamente significativas em relação à percepção 
Figura 1

Áreas de abrangência das oito Gerências Distritais (GD) de Saúde do Município de Porto Alegre, Rio Grande do Sul, Brasil, 2011.

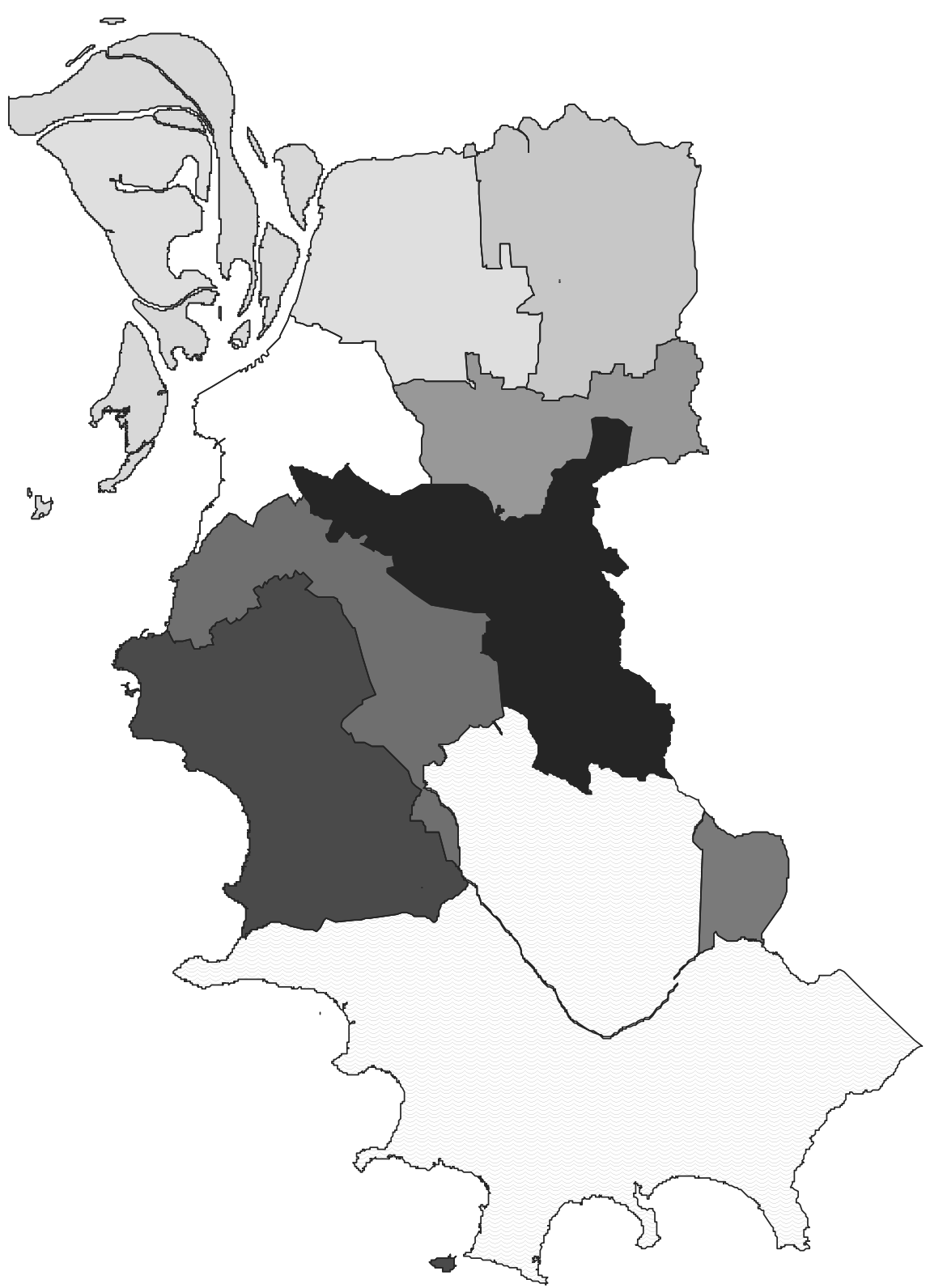

GD Centro

GD Nordeste/Humaitá-Navegantes/llhas

GD Norte/Eixo-Baltazar

GD Leste Nordeste

GD Glória/Cruzeiro/Cristal

GD Sul/Centro-Sul

GD Partenon/Loma

GD Restinga/Extremo-Sul
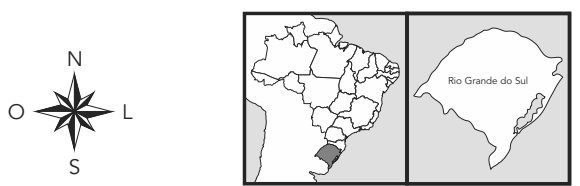
Indicadores de saúde contidos no relatório SSA2 do Sistema de Informação da Atenção Básica (SIAB) *, 2011.

\begin{tabular}{|c|c|}
\hline Indicador & Indicador em saúde \\
\hline 1 & Gestantes acompanhadas no mês \\
\hline 2 & Gestantes que estão com vacinas em dia \\
\hline 3 & Gestantes que tiveram consultas de pré-natal no mês \\
\hline 4 & Gestantes que iniciaram o pré-natal no 1o trimestre \\
\hline 5 & Gestantes com menos de 20 anos de idade cadastradas \\
\hline 6 & Recém-nascido com peso $<2.500 \mathrm{~g}$ \\
\hline 7 & Crianças de 0 a 3 meses que estão em aleitamento materno exclusivo \\
\hline 8 & Crianças de 0 a 11 meses com vacinas em dia \\
\hline 9 & Crianças de 0 a 11 meses que estão desnutridas \\
\hline 10 & Crianças de 12 a 23 meses com vacinas em dia \\
\hline 11 & Crianças de 12 a 23 meses que estão desnutridas \\
\hline 12 & Crianças menores de 2 anos de idade que tiveram diarreia \\
\hline 13 & Crianças menores de 2 anos de idade que tiveram episódio de infecção respiratória aguda \\
\hline 14 & Pessoas com diagnóstico de diabetes acompanhada \\
\hline 15 & Prevalência de diabetes na população acompanhada \\
\hline 16 & Pessoas com diagnóstico de hipertensão acompanhado \\
\hline 17 & Prevalência de hipertensão na população acompanhada \\
\hline 18 & Pessoas com diagnóstico de tuberculose acompanhado \\
\hline 19 & Pessoas com diagnóstico de hanseníase acompanhado \\
\hline 20 & Crianças menores de cinco anos de idade hospitalizadas por pneumonia \\
\hline 21 & Crianças menores de cinco anos de idade hospitalizadas por desidratação \\
\hline 22 & Pessoas hospitalizadas por abuso de álcool \\
\hline 23 & Pessoas hospitalizadas por complicações em virtude da diabetes \\
\hline 24 & Pessoas com internação em hospital psiquiátrico \\
\hline
\end{tabular}

* Dados disponibilizados pela Secretaria Municipal de Saúde de Porto Alegre (Situação de saúde e o acompanhamento das famílias. Acessado em 18/Jan/2010).

dos profissionais quanto à classificação da situação de saúde. Para isso o teste ANOVA de medidas repetitivas comparou as médias dos valores apontados pelos pesquisados e foi comprovada a mudança na percepção da situação de saúde por parte dos profissionais ao longo dos três momentos da pesquisa.

Ocorreram variações importantes nas médias com relação à percepção dos profissionais na maioria dos indicadores estudados. No entanto, houve maior diferenciação no caso das doenças crônicas, especialmente, hipertensão arterial e diabetes mellitus, como mostra a Tabela 2. A classificação da situação de saúde para doenças crônicas no momento I apresentou uma condição mais alta (mais positiva) do que no momento II e no momento III quando introduzido o SIABg em que a classificação média foi mais baixa (menos positiva).

A estratificação da análise entre os três momentos da pesquisa evidencia que quando os profissionais fazem o uso do SIABg (momento III) comparado com o inicial empírico (momento I) e o SIAB tradicional (momento II), aumenta a diferença de sua percepção da situação de saúde medida pelo teste F. Além disso, em várias das situações de saúde o SIAB tradicional não mostrou diferença significativa na percepção em relação ao momento inicial, mas o uso do modelo georreferenciado provocou alteração quando comparado com o empírico em nove dos 24 $(37,5 \%)$ indicadores estudados.

\section{Discussão}

A pesquisa evidencia que o georreferenciamento, agregado a um SIS nas microáreas dos ACS, promove outra percepção da situação de saúde da população. Os profissionais puderam visualizar a distribuição espacial das doenças o que permite que as unidades de saúde conheçam melhor as 
Figura 2

Parte do relatório SSA2 do Sistema de Informação da Atenção Básica (SIAB) *, que mostra a situação de saúde e acompanhamento georreferenciados das famílias (SSA2g) da Unidade de Saúde da Família (USF) D, 2011.

2a) Número de crianças de 0 a 11 meses e 29 dias

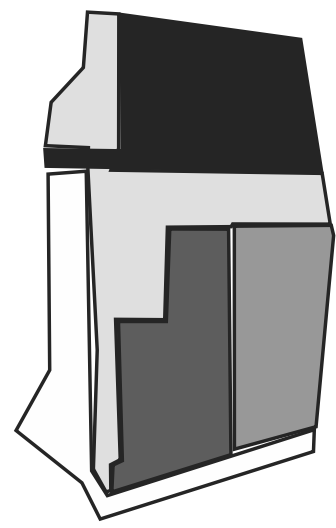

2b) Número de crianças de 0 a 11 meses e 29 dias desnutridas
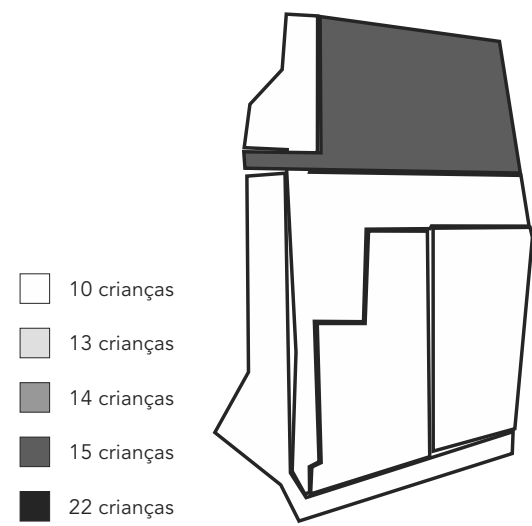

0 crianças

1 criança 2c) Porcentagem de crianças de 0 a 11 meses e 29 dias desnutridas

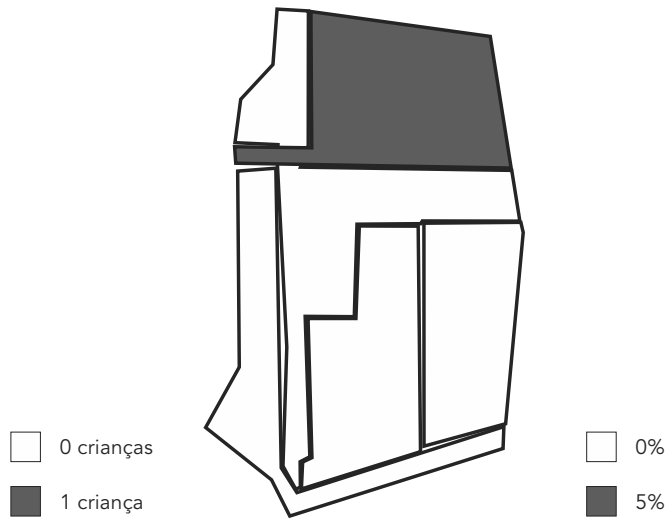

2e) Número de crianças de 12 a 23 meses e 29 dias com vacinas em dia

2f) Porcentagem de crianças de 12 a 23 meses e 29 dias com vacinas em dia
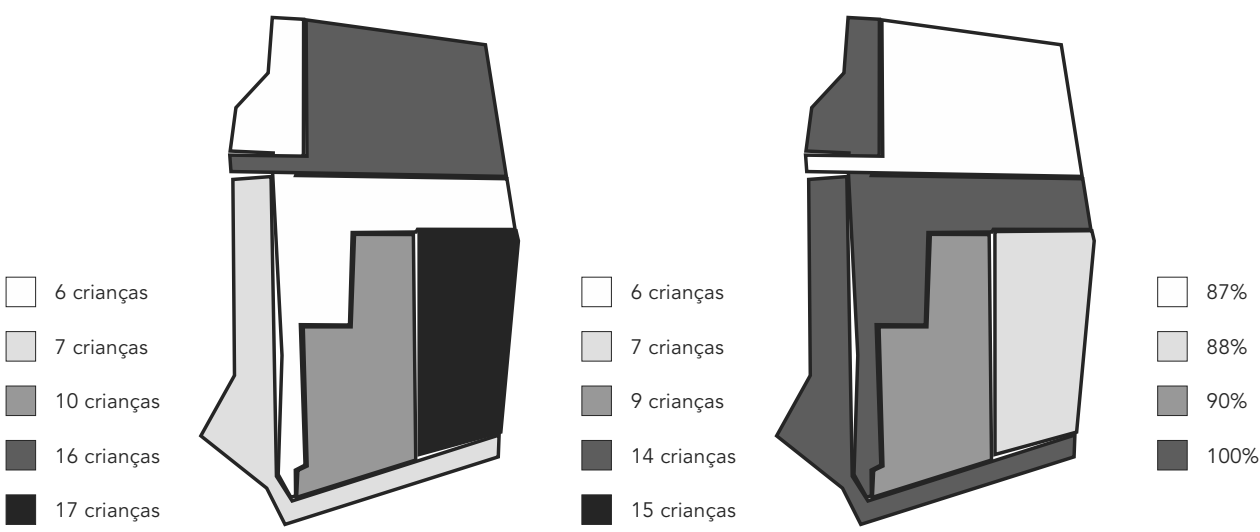

* Dados disponibilizados pela Secretaria Municipal de Saúde de Porto Alegre (Situação de saúde e o acompanhamento das famílias. Acessado em 18/Jan/2010).

características da população adscrita, bem como desenvolvam programas e projetos viáveis e bem direcionados frente aos diagnósticos levantados. Estudar aspectos geográficos no uso dos serviços de saúde gera discussões que poderiam estar sendo negligenciadas, sendo assim métodos de análise espacial no campo da saúde coletiva para o monitoramento e avaliação dos serviços de saúde podem ser positivos 26,27.

Uma das principais diretrizes atuais do $\mathrm{Mi}$ nistério da Saúde é executar a gestão pública com base na indução, monitoramento e avaliação de processos e resultados mensuráveis, garantindo acesso e qualidade da atenção em saúde a toda a população, para isso, em 2011, é instituído o Programa para Melhoria do Acesso e Qualidade da Atenção Básica (PMAQ) pela Portaria GM no $1.654 / 201128$. O principal objetivo é induzir a ampliação do acesso e a melhoria da qualidade da atenção primária em saúde, com garantia de um padrão de qualidade, de maneira a permitir maior transparência e efetividade das ações em saúde 29 . 
Tabela 2

Médias, desvios-padrão (DP) e teste de contraste com ANOVA de medidas repetitivas que identifica a modificação na percepção da situação de saúde da população das unidades da Estratégia Saúde da Família (ESF) de uma Gerência Distrital de Saúde de Porto Alegre, Rio Grande do Sul, Brasil, entre os momentos I, II e III, 2011.

\begin{tabular}{|c|c|c|c|}
\hline Indicador de saúde & $\begin{array}{l}\text { Momento I } \\
\text { Média (DP) }\end{array}$ & $\begin{array}{l}\text { Momento II } \\
\text { Média (DP) }\end{array}$ & $\begin{array}{l}\text { Momento III } \\
\text { Média (DP) }\end{array}$ \\
\hline 1. Gestantes acompanhadas no mês & $3,90(0,85)$ & $3,97(0,66)$ & $4,40(0,96)$ \\
\hline Momento I versus momento II versus momento III & & $F(2)=6,61 ; p<0,05$ & \\
\hline Momento I versus momento II & & $F(1)=1,04 ; p=0,31$ & \\
\hline Momento I versus momento III & & $F(1)=9,28 ; p<0,05$ & \\
\hline Momento II versus momento III & & $F(1)=11,46 ; p<0,05$ & \\
\hline 2. Gestantes com menos de 20 anos de idade cadastradas & $3,61(1,20)$ & $3,29(0,97)$ & $2,65(0,83)$ \\
\hline Momento I versus momento II versus momento III & & $F(2)=14,38 ; p<0,001$ & \\
\hline Momento I versus momento II & & $F(1)=1,93 ; p=0,17$ & \\
\hline Momento I versus momento III & & $F(1)=27,85 ; p<0,001$ & \\
\hline Momento II versus momento III & & $F(1)=10,32 ; p<0,05$ & \\
\hline 3. Recém-nascido com peso menor que 2.500 gramas & $3,72(1,14)$ & $3,86(1,06)$ & $3,31(0,70)$ \\
\hline Momento I versus momento II versus momento III & & $F(2)=5,15 ; p<0,05$ & \\
\hline Momento I versus momento II & & $F(1)=0,94 ; p=0,34$ & \\
\hline Momento I versus momento III & & $F(1)=4,13 ; p<0,05$ & \\
\hline Momento II versus momento III & & $F(1)=9,24 ; p<0,05$ & \\
\hline 4. Crianças de 0 a 3 meses que estão em aleitamento materno exclusivo & $3,38(0,92)$ & $3,31(0,77)$ & $2,61(0,81)$ \\
\hline Momento I versus momento II versus momento III & & $F(2)=11,51 ; p<0,001$ & \\
\hline Momento I versus momento II & & $F(1)=0,08 ; p=0,78$ & \\
\hline Momento I versus momento III & & $F(1)=20,27 ; p<0,001$ & \\
\hline Momento II versus momento III & & $F(1)=16,43 ; p<0,001$ & \\
\hline 5. Crianças de 0 a 11 meses com vacinas em dia & $3,81(0,81)$ & $3,86(0,87)$ & $3,27(0,75)$ \\
\hline Momento I versus momento II versus momento III & & $F(2)=11,84 ; p<0,001$ & \\
\hline Momento I versus momento II & & $F(1)=0,31 ; p=0,58$ & \\
\hline Momento I versus momento III & & $F(1)=14,46 ; p<0,001$ & \\
\hline Momento II versus momento III & & $F(1)=13,58 ; p<0,001$ & \\
\hline 6. Crianças de 0 a 11 meses que estão desnutridas & $4,11(1,06)$ & $3,79(1,26)$ & $4,47(0,73)$ \\
\hline Momento I versus momento II versus momento III & & $F(2)=6,37 ; p<0,05$ & \\
\hline Momento I versus momento II & & $F(1)=1,26 ; p=0,27$ & \\
\hline Momento I versus momento III & & $F(1)=2,77 ; p<0,05$ & \\
\hline Momento II versus momento III & & $F(1)=11,34 ; p<0,05$ & \\
\hline 7. Crianças menores de 2 anos de idade que tiveram diarreia & $3,45(1,40)$ & $3,65(1,05)$ & $4,29(0,73)$ \\
\hline Momento I versus momento II versus momento III & & $F(2)=8,44 ; p<0,05$ & \\
\hline Momento I versus momento II & & $F(1)=1,00 ; p=0,32$ & \\
\hline Momento I versus momento III & & $F(1)=13,13 ; p<0,05$ & \\
\hline Momento II versus momento III & & $F(1)=11,58 ; p<0,05$ & \\
\hline $\begin{array}{l}\text { 8. Crianças menores de } 2 \text { anos de idade que tiveram algum episódio de } \\
\text { infecção respiratória aguda }\end{array}$ & $3,20(0,92)$ & $3,54(1,06)$ & $3,95(0,83)$ \\
\hline Momento I versus momento II versus momento III & & $F(2)=7,91 ; p<0,05$ & \\
\hline Momento I versus momento II & & $F(1)=3,34 ; p=0,07$ & \\
\hline Momento I versus momento III & & $F(1)=15,14 ; p<0,001$ & \\
\hline Momento II versus momento III & & $F(1)=4,90 ; p<0,05$ & \\
\hline 9. Pessoas com diagnóstico de diabetes acompanhada & $3,65(0,88)$ & $3,29(0,66)$ & $2,84(1,14)$ \\
\hline Momento I versus momento II versus momento III & & $F(2)=6,59 ; p<0,05$ & \\
\hline Momento I versus momento II & & $F(1)=4,43 ; p<0,05$ & \\
\hline Momento I versus momento III & & $F(1)=13,12 ; p<0,05$ & \\
\hline Momento II versus momento III & & $F(1)=8,00 ; p<0,05$ & \\
\hline
\end{tabular}

(continua) 
Tabela 2 (continuação)

\begin{tabular}{|c|c|c|c|}
\hline Indicador de saúde & $\begin{array}{l}\text { Momento I } \\
\text { Média (DP) }\end{array}$ & $\begin{array}{l}\text { Momento II } \\
\text { Média (DP) }\end{array}$ & $\begin{array}{l}\text { Momento III } \\
\text { Média (DP) }\end{array}$ \\
\hline 10. Prevalência de diabetes na população acompanhada & $4,38(1,93)$ & $3,36(0,91)$ & $2,47(0,82)$ \\
\hline Momento I versus momento II versus momento III & & $F(2)=33,13 ; p<0,001$ & \\
\hline Momento I versus momento II & & $F(1)=11,20 ; p<0,05$ & \\
\hline Momento I versus momento III & & $F(1)=41,63 ; p<0,001$ & \\
\hline Momento II versus momento III & & $F(1)=24,60 ; p<0,001$ & \\
\hline 11. Pessoas com diagnóstico de hipertensão acompanhado & $3,65(0,91)$ & $3,27(0,65)$ & $2,61(1,31)$ \\
\hline Momento I versus momento II versus momento III & & $F(2)=8,36 ; p<0,05$ & \\
\hline Momento I versus momento II & & $F(1)=5,57 ; p<0,05$ & \\
\hline Momento I versus momento III & & $F(1)=15,92 ; p<0,001$ & \\
\hline Momento II versus momento III & & $F(1)=14,71 ; p<0,001$ & \\
\hline 12. Prevalência de hipertensão na população acompanhada & $4,40(1,90)$ & $3,34(0,91)$ & $2,54(0,69)$ \\
\hline Momento I versus momento II versus momento III & & $F(2)=23,99 ; p<0,001$ & \\
\hline Momento I versus momento II & & $F(1)=12,59 ; p<0,05$ & \\
\hline Momento I versus momento III & & $F(1)=35,49 ; p<0,001$ & \\
\hline Momento II versus momento III & & $F(1)=20,24 ; p<0,001$ & \\
\hline 13. Pessoas com diagnóstico de tuberculose acompanhado & $3,93(1,12)$ & $4,50(1,02)$ & $4,40(0,90)$ \\
\hline Momento I versus momento II versus momento III & & $F(2)=3,84 ; p<0,05$ & \\
\hline Momento I versus momento II & & $F(1)=6,67 ; p<0,05$ & \\
\hline Momento I versus momento III & & $F(1)=7,20 ; p<0,05$ & \\
\hline Momento II versus momento III & & $F(1)=0,00 ; p=1,00$ & \\
\hline 14. Pessoas hospitalizadas por abuso de álcool & $3,47(1,38)$ & $3,97(1,30)$ & $4,04(0,83)$ \\
\hline Momento I versus momento II versus momento III & & $F(2)=3,29 ; p<0,05$ & \\
\hline Momento I versus momento II & & $F(1)=3,65 ; p=0,06$ & \\
\hline Momento I versus momento III & & $F(1)=6,73 ; p<0,05$ & \\
\hline Momento II versus momento III & & $F(1)=0,09 ; p=0,76$ & \\
\hline
\end{tabular}

Com essa necessidade identificada pelo Ministério da Saúde de qualificar a avaliação da atenção primária em saúde pelos próprios serviços, uma melhor visualização dos dados da situação de saúde no território pode ser fundamental, pois a pesquisa mostrou, no caso das doenças crônicas (hipertensão e a diabetes), uma inversão do entendimento das condições de saúde na população e da prevalência da doença. Conseguiram-se definir com maior precisão, por exemplo, prioridades das famílias. Usar um modelo de distribuição espacial para variáveis individuais tornou factível para os profissionais identificar áreas onde existe um risco aumentado para os indivíduos com doenças crônicas, o que pode ser um instrumento valioso que possibilite identificar e controlar fatores individuais 30 . Esse modelo de distribuição espacial localiza com exatidão situações de saúde e pode-se propor ações direcionadas para as comunidades.

Para tanto, o georreferenciamento dos dados da situação da saúde da população pode ser uma ferramenta importante no processo de autoava- liação das ações em atenção primária em saúde. Em 2011 o Ministério da Saúde reforça isso com a disponibilização do instrumento denominado Autoavaliação para a Melhoria do Acesso e da Qualidade da Atenção Básica (AMAQ) ${ }^{31}$ entendido como ponto de partida da fase de desenvolvimento da qualificação dos processos de avaliação, uma vez que deflagra a identificação e reconhecimento das dimensões positivas e também problemáticas do trabalho nas equipes, o que o SIS georreferenciado mostrou como instrumento interessante. Isso porque os dados dessa forma passaram a ter melhor sentido e principalmente significado, potencializando e facilitando mudanças e aprimoramentos nos serviços 31,32,33.

Para tanto, a autonomia pode ser mais bem aproveitada a partir do conjunto de ações no momento em que o processo de territorialização está interligado ao SIS e esse com a função de ser organizador do processo de trabalho das equipes. No entanto, a territorialização, muitas vezes, está reduzida ao conceito de espaço, e assim pas- 
sa a ser utilizada de forma meramente administrativa para a gestão física dos serviços de saúde, negligenciando-se o potencial como ferramenta de gestão local 30,32.

A diferença demonstrada na pesquisa para definição da situação da saúde pelos profissionais pode indicar que a equipe não tem tempo para observar o resultado do seu trabalho. $\mathrm{Ou}$ ainda, que as ferramentas dessa análise, os SIS, não estão sendo suficientemente objetivas para as funções que foram criadas. A maioria dos SIS trazem seus dados na forma de tabelas repletas de números (os consolidados), que são importantes para um diagnóstico situacional do estado de saúde das pessoas de quem eles falam, mas pouco práticos e estimuladores para os que usam.

A pesquisa mostra também que a utilização ou não dos sistemas de informação apresentada de forma tradicional não mostrou diferença significativa na percepção da situação de saúde de cada uma das comunidades estudadas. Ou seja, a forma como eles são apresentados não pode ser considerada como instrumento de fácil visualização e principalmente interpretação da situação de saúde para o processo de trabalho em atenção primária em saúde. Por isso, os profissionais preferem trabalhar na lógica do empirismo, ou seja, com o que provém unicamente da experiência prática e subjetiva de cada um. E assim os SIS não são aproveitados na sua potencialidade.

Desse modo, tendo que trabalhar sem ter instrumentos ou sistemas práticos para fazer um planejamento baseado em evidências, os profissionais acabam por conduzir suas ações diárias pela lógica da experiência prática, intuitiva e, sobretudo, subjetiva. Não raramente, esses têm o seu tempo consumido por ações de pronto atendimento, contrário ao preconizado pela Política Nacional de Atenção Básica, Portaria GM no $2.488 / 2011^{3}$. Agindo assim, passam a focalizar a sequela da doença e não as causas, pois não identificam áreas ou situações de risco com os SIS disponíveis.

Os SIS não podem exigir gastos de energia para serem analisados e compreendidos, pois a informação é o dado trabalhado, tratado, útil, com valor significativo atribuído ou agregado, com um sentido, acima de tudo, natural e lógico para quem usa a informação ${ }^{34}$. Para tanto é necessário que haja investimentos tanto na educação permanente dos profissionais, como também nas discussões e interpretações dos dados coletados. No entanto, a preocupação maior dos profissionais em relação aos SIS se concentra no preenchimento das fichas e não na análise das informações que ele são capazes de fornecer para a realização da programação local 34 .
Com relação à formação dos profissionais, a educação permanente, bem como a avaliação constante das dificuldades de utilização dos SIS, devem ter apoio das Gestões Municipais como traz a Política Nacional de Educação Permanente $35,36,37$. Isso subsidiará os profissionais para poderem enfrentar as dificuldades identificadas na operacionalização dessa tecnologia. Não basta ter acesso só aos manuais e cobrar a operacionalização, é preciso investir em capacitações.

\section{Conclusão}

A introdução de indicadores de saúde georreferenciados no processo de trabalho de USF agregou uma nova tecnologia que permite aos profissionais otimizarem o seu tempo de trabalho. A forma de apresentação dos dados desse sistema permitiu melhor descrição e principalmente detalhamento de forma territorializada. O SIAB não tem alcançado, na íntegra, o objetivo de ser um instrumento de reorganização das práticas de trabalho. Porém, o georreferenciamento dos sistemas de informação proporcionou maior clareza e rapidez para desvendar detalhes, antes de pouca importância, em cada uma das microáreas que compõem as USF.

A disposição espacial permite melhor associação e consequente interpretação dos indicadores de saúde, objetos para determinação de comorbidades em saúde que acometem os coletivos das pessoas. Por isso, o georreferenciamento vai ao encontro das melhores práticas de vigilância em saúde a nível local, pois permite uma tomada de decisão mais adequada às realidades sanitárias e epidemiológicas. Sendo assim, possibilita a caracterização e a identificação de situações de saúde, através de um diagnóstico situacional mais preciso, o que gera subsídios sólidos para o planejamento.

Entre as limitações dessa investigação, devese considerar o viés de seleção da amostra selecionada que foi escolhida em uma única GD de Porto Alegre por conveniência. Além disso, há dificuldade de comparações com outras investigações, visto que o instrumento para coleta dos dados é original e não validado. Por último, é possível que os participantes integrantes da amostra estivessem mais motivados e dispostos a modificar a sua percepção com relação à situação de saúde das pessoas e famílias que os não participantes.

O georreferenciamento dos sistemas de informação é mais eficiente para gerenciamento do processo de trabalho e um importante auxílio para respaldar as ações em atenção primária em saúde que se articulam com as instâncias de 
controle social e a gestão municipal. As novas tecnologias de informação que impactam diretamente a capacidade de reconhecer antecipadamente os problemas, como pode ser considerado o georreferenciamento dos dados, permitem uma agregação de dados sobre a situação de saúde da população e promovem agilidade e simples manejo das informações. Esse instrumento propicia melhor identificação de áreas de vulnerabilidade e assim torna mais eficiente o processo de tomada de decisão, gerando serviços mais resolutivos e equânimes.

Por fim, é necessário que os municípios criem ou ampliem as equipes que gerenciarão os recursos informacionais, uma limitação para que o georreferenciamento se concretize. Além disso, é necessário que as equipes se responsabilizem pela qualificação das suas ações e isso passa necessariamente pela problematização dos dados que mostram a situação de saúde das pessoas sob a responsabilidade das unidades de saúde, juntos à gestão e ao controle social. Desse modo, os SIS serão colocados na agenda de trabalho de cada um dos serviços de saúde para que então se tornem uma ferramenta norteadora das ações. Para o município tem-se a possibilidade de estabelecer um pacto para a diminuição de custos ao longo do tempo no momento que permite que os trabalhadores possam fazer melhor adequação das práticas.

\section{Resumen}

En la atención primaria de salud poco han valido la pena los sistemas de información para evaluar el estado de salud de la población, debido a la dificultad de entender los informes; al tratarse de definiciones genéricas para las acciones habituales de los resultados empíricos. El objetivo de este estudio es evaluar si los indicadores de información georreferenciada pueden ser una tecnología que mejore la identificación de la situación de salud para las personas que ayuden en la planificación de acciones. Para ello, se aplicó un cuestionario por parte de ocho equipos profesionales en tres etapas: la primera, antes de la lectura de los informes del sistema de información, la segunda y la tercera lectura, después de usar información georreferenciada.
Los resultados mostraron diferencias significativas en la clasificación del estado de salud al utilizar georreferenciación en comparación con períodos anteriores ( $p<0,05)$. La geocodificación facilitó el análisis de la situación de salud, proporcionando un mejor monitoreo de los procesos de trabajo. Por último, se utilizaron puntos para la racionalización de las acciones y la posible calificación de la atención sanitaria. Sugerimos el uso de georreferenciación regularmente para que se convierta en una herramienta eficaz en las acciones de orientación.

Salud de la Familia; Sistemas de Información

Geográfica; Evaluación en Salud 


\section{Colaboradores}

Todos os autores participaram substancialmente de todas as etapas de elaboração do artigo.

\section{Referências}

1. White PL. Information for health care: an epidemiological perspective. Inquiry 1980; 17:296-312.

2. Figueiredo LA, Pinto IC, Marciliano CSM, Souza MF, Batista Guedes AA. Análise da utilização do SIAB por quatro equipes da estratégia saúde da família do município de Ribeirão Preto, SP. Cad Saúde Colet (Rio J.) 2010; 18:418-23.

3. Ministério da Saúde. Portaria MS/GM no 2.488, de 21 de outubro de 2011. Aprova a Política Nacional de Atenção Básica, estabelecendo a revisão de diretrizes e normas para a organização da Atenção Básica, para a Estratégia Saúde da Família (ESF) e o Programa de Agentes Comunitários de Saúde (PACS). Diário Oficial da União 2011; 24 out.

4. Mishima SM, Villa TCS. A enfermagem e o sistema de saúde. Rev Latinoam Enferm 1996; 4:1-2.

5. Pereira MPB, Barcellos C. O território no Programa de Saúde da Família. Revista Brasileira de Geografia Médica e da Saúde 2006; 2:47-55.

6. Milsoczky MC. Distrito sanitário: desafio de descentralizar com democracia. Saúde Debate 1991; 33:54-60.

7. Pereira MG. Epidemiologia: teoria e prática. Rio de Janeiro: Editora Guanabara Koogan; 1995.

8. Barros MBA. Epidemiologia e superação das iniqüidades em saúde. In: Barata RB, Marreto ML, Almeida Filho N, organizadores. Equidade e saúde, contribuições da epidemiologia. Rio de Janeiro: Editora Fiocruz/ABRASCO; 1997.
9. Ministério da Saúde. Sistema de Informação da Atenção Básica - SIAB: indicadores 2005. Brasília: Ministério da Saúde; 2005.

10. Mishima SM, Oliveira TH, Pinto IC. O trabalho do enfermeiro na organização dos serviços de saúde e sua inserção no departamento de informática da SMS-RP. Rev Latinoam Enferm 1999; 7:13-20.

11. Ministério da Saúde. Manual do Sistema de Informação da Atenção Básica. Brasília: Ministério da Saúde; 2000.

12. Moraes IHLM, Santos SRRFR. Informações para a gestão do SUS: necessidades e perspectivas. Inf Epidemiol SUS 2001; 10:49-56.

13. Faria HP, Werneck MAF, Santos MA, Teixeira PF. Processo de trabalho em saúde. 2a Ed. Belo Horizonte: Núcleo de Educação em Saúde Coletiva, Faculdade de Medicina, Universidade Federal de Minas Gerais/Editora Coopmed; 2009.

14. Oliveira HM, Pires TO, Parente RCP. As relações de poder na Estratégia de Saúde da Família sob o enfoque da teoria de Hannah Arendt. Revista Saúde \& Transformação Social 2011; 1:17-26.

15. Silva IZQJ, Trad LAB. O trabalho em equipe no PSF: investigando a articulação técnica e a interação entre os profissionais. Interface Comum Saúde Educ 2005; 9:25-38.

16. Capucci PF, Martins CL, Ianni AMZ, Carneiro Jr. N, Bógus CM. Um olhar sobre o VI Congresso Paulista de Saúde Pública. Saúde Soc 1999; 8:109-23. 
17. Monken M, Barcellos C. Vigilância em saúde e território utilizado: possibilidades teóricas e metodológicas. Cad Saúde Pública 2005; 21:898-906.

18. Barcellos C, Ramalho WM, Gracie R, Magalhães MAFM, Fontes MP, Skaba D. Georreferenciamento de dados de saúde na escala submunicipal: algumas experiências no Brasil. Epidemiol Serv Saúde 2008; 17:59-70.

19. Kadt E, Tasca R. Promovendo a equidade: um novo enfoque com base no setor da saúde. São Paulo: Editora Hucitec/Salvador: Cooperativa Italiana em Saúde; 1993.

20. Barcellos C, Sabroza PC, Peiter P, Rojas LI. Organização espacial, saúde e qualidade de vida: análise espacial e uso de indicadores na avaliação de situações de saúde. Inf Epidemiol SUS 2002; 11: 129-38.

21. Ferreira SMG, Azevedo PCV. Sistema de informação para tomada de decisões em saúde - SINTOMAS: sistema de informação geográfico para sistemas locais de saúde. In: Mendes EV, organizador. A organização da saúde no nível local. São Paulo: Editora Hucitec; 1998. p. 175-83.

22. Chiesa AM, Westphal MF, Kashiwagi NM. Geoprocessamento e a promoção da saúde: desigualdades sociais e ambientais em São Paulo. Rev Saúde Pública 2002; 36:559-67.

23. Piovesan MF, Padrão MVV, Dumont UM, Gondim GM, Flores O, Pedrosa JI, et al. Vigilância sanitária: uma proposta de análise dos contextos locais. Rev Bras Epidemiol 2005; 8:83-95.

24. Likert R. A technique for the measurement of attitudes. Arch Psychol 1932; 140:1-50.

25. Field A. Descobrindo a estatística usando SPSS Porto Alegre: Editora Artmed; 2009.

26. Bailey TC. Spatial statistics methods in health. Cad Saúde Pública 2001; 17:1083-98.

27. Elliott P, Wartenberg D. Spatial epidemiology: current approaches and future challenges. Environ Health Perspect 2004; 112:998-1006.
28. Departamento de Atenção Básica, Secretaria de Atenção à Saúde, Ministério da Saúde. Programa Nacional de Melhoria do Acesso e da Qualidade da Atenção Básica (PMAQ): manual instrutivo. Brasília: Ministério da Saúde; 2012.

29. Frias PG, Samico I, Felisberto E, Figueiró AC. Atributos da qualidade em saúde. In: Samico I, Felisberto E, Figueiró AC, Frias PG, organizadores. Avaliação em saúde: bases conceituais e operacionais. Rio de Janeiro: Medbook; 2010. p. 43-56.

30. Carvalho MS, Souza-Santos R. Análise de dados espaciais em saúde pública: métodos, problemas, perspectivas. Cad Saúde Pública 2005; 21:361-78.

31. Departamento de Atenção Básica, Secretaria de Atenção à Saúde, Ministério da Saúde. Autoavaliação para a melhoria do acesso e da qualidade da atenção básica - AMAQ. Brasília: Ministério da Saúde; 2011.

32. Hartz ZMA, Vieira-da-Silva LM. Avaliação em saúde: dos modelos teóricos a prática na avaliação de programas e sistemas de saúde. Rio de Janeiro: Editora Fiocruz; 2008.

33. Levitt C, Hilts L. Quality book of tools. Hamilton: MC Master Innovation Press; 2010.

34. Rezende DA. Planejamento de sistemas de informação e informática. São Paulo: Editora Atlas; 2003.

35. Freitas FP, Pinto IC. Percepção da Equipe de Saúde da Família sobre a utilização do Sistema de Informação da Atenção Básica - SIAB. Rev Latinoam Enferm 2005; 13:547-54.

36. Ministério da Saúde. Portaria GM no 1.996 de 20 de agosto de 2007. Dispõe sobre as diretrizes para implantação da Política Nacional de Educação Permanente em Saúde. Diário Oficial da União 2007; 22 ago.

37. Moraes IHS. Informações em Saúde: da prática fragmentada ao exercício da cidadania. Rio de Janeiro: ABRASCO/São Paulo: Editora Hucitec; 1994.

Recebido em 07/Set/2012

Versão final reapresentada em 2/Abr/2013

Aprovado em 26/Jun/2013 